\title{
Correspondence
}

\section{Renal subcapsular versus portal vein site for islet transplantation in mice}

\section{Dear Sir,}

In their article on growth conditions for transplanted mouse pancreatic islet cells, Mellgren et al. [1] demonstrate superiority of the renal subcapsular space as a site for proliferation of pancreatic islet isografts in the mouse model.

Interestingly, studies from our laboratory demonstrated the portal vein provided a privileged site for murine islet allografts when compared to the renal subcapsular space [2]. In these studies, islets were transplanted to either the portal vein or renal subcapsular space of streptozotocin-induced diabetic mice in two different strain combinations. In the first combination, B10.BR islets $\left(\mathrm{H}-2^{\mathrm{k}}\right)$ were transplanted into $\mathrm{C} 57 \mathrm{~B} / 6$ recipients $\left(\mathrm{H}-2^{\mathrm{b}}\right)$. These strains differ at the $\mathrm{K}$, I and $\mathrm{D}$ regions for the entire $\mathrm{H}-2$ haplotype. In the other combination, B10.A2R ( $\mathrm{a} / \mathrm{b}$ haplotype) mice were recipients of allografts from B10.AQR donors ( ${ }^{y} 1$ haplotype). This strain combination is identical at the $\mathrm{I}$ region but differs at both the $\mathrm{K}$ and $\mathrm{D}$ region.

The results of the experiments were similar in both donor-recipient strain combinations. In both of these combinations, the frequency of rejection was lower and the median functional survival time was longer for grafts at the portal vein site than the subcapsular site. We concluded that the portal vein was a better site than the kidney capsule for islet transplantation in mice [2], just the opposite of the conclusion of Mellgren et al [1].

This discrepancy could relate to the fact that our transplants were allografts, while those of Mellgren et al. [1] were isografts. Mellgren et al [1] cite the possibility that islet isografts placed in the renal subcapsular space have a better supply of well-oxygenized blood than islets transplanted to the other sites, perhaps due to differences in the rates of neovascularization. To support this contention, they report the finding of numerous mast cells in and beneath the kidney capsule close to the islet isografts, suggestive of an active angiogenesis in this area [3]. Our finding of higher rates of rejection of islet allografts transplanted to the renal subcapsular position are consistent with this finding, in that active angiogenesis would provide an inflammatory milieu leading to rejection of the transplanted islets. Although the kidney capsule might offer better growth conditions for transplanted mouse pancreatic isografts, these better conditions may be responsible for the higher rates of rejection when islet allografts are transplanted to this position.

Yours sincerely,

P. G. Stock, P. Gores and D. E. R. Sutherland

\section{References}

1. Mellgren A, Schnell Landstrom AH, Petersson B, Andersson A (1986) The renal subcapsular site offers better growth conditions for transplanted mouse pancreatic islet cells than the liver or spleen. Diabetologia 29: 670-672

2. Gores PF, Rabe F, Sutherland DER (1987) Prolonged survival of intraportal versus subrenal capsular transplanted islet allografts. Transplantation (in press)

3. Folkman J (1985) Toward an understanding of angiogenesis: search and discovery. Perspect Biol Med 29:1-36

Dr. P. Gores

Box 31

University of Minnesota Hospitals

420 Delaware Street, S.E.

Minneapolis, MN 55455, USA

\section{Evidence for a specific heart disease in Type 1 (insulin-dependent) diabetic patients?}

\section{Dear Sir,}

Fisher et al. [1] recently reported evidence for a specific heart disease in Type 1 diabetic patients.

They performed radionuclide ventriculography during dynamic exercise, during isometric exercise and during cold pressor testing in 63 asymptomatic Type 1 diabetic patients. Confirming previous reports [2], they found significantly less increase in ejection fraction (EF) during dynamic exercise as compared to normal control subjects. The negative results concerning isometric exercise and cold pressor testing are also compatible with the general impression of insensitivity of these tests.

In the male group an increase of EF less than 5\% during dynamic exercise was considered abnormal, as this response has been found to be a reliable predictor of coronary artery disease in earlier studies. Nine out of 35 male patients and 1 out of 25 normal men had an abnormal test. Two of those patients were investigated by coronary arteriography and endomyocardial biopsy and had normal coronary arteries and normal ventriculography. Unfortunately, detailed data are not given on the endomyocardial biopsy. One of those men had a duration of disease of 11 years and no signs of nephropathy or retinopathy, which usually accompany preclinical and clinical signs of cardiomyopathy in diabetic patients [3]. Therefore, we suspect the stress test to be falsely positive in this patient.

In the group of female patients $16 \%$ of the normal control female subjects had an increase in EF of less than 5\% during dynamic exercise. Therefore, the authors redefined the pathological test result as a failure to rise or a fall in EF on dynamic exercise. This may not be justifiable, since a more likely explanation for the result is that the female subjects had not been exercised to a sufficient degree.

Thus, the authors base their conclusion on a secondarily defined test result in the female group ( $75 \%$ of the patients invasively investigated) and on two male patients, in whom a false positive test result may be expected in one.

On the basis of earlier studies [4], we agree with the authors that a specific diabetic heart disease is likely to exist in man, but we do not think its existence has been supported by their paper.

Yours sincerely,

L. Thuesen and J.Sandahl Christiansen 